\title{
The optical microscopic observation of frictional interface between high strength gels and a glass ball by ball-on-disk method
}

\author{
Naoya Yamada ${ }^{1} \cdot$ Masato Wada $^{2} \cdot$ Masaru Kawakami $^{1} \cdot$ Hidemitsu Furukawa $^{1}$ (D)
}

Received: 3 July 2019/Accepted: 18 July 2019/Published online: 3 September 2019

(C) The Author(s) 2019

\begin{abstract}
The polymer gels with the properties of high strength and low friction show promise as the ideal materials for a living body-like soft robot joint. To date, the relationship between contact area and friction behavior of hydrogels has been revealed from the observation of flat frictional surface. Here, we designed the microscopic observation system for the friction surface of soft materials under deformation condition. A specially arranged ball-on-disk measuring part was combined with inverted microscope to observe the frictional interface during the friction. Both of transparent glass ball and moving glass stage were employed in this instrument and the microscope camera was thereby able to take the moving picture of frictional interface imaged by the refractive index differences between a soda-lime glass ball, transparent hydrogels and air. By using this customized measuring instrument, when water was not rich on the friction surface, the moving pictures of the frictional interface between glass ball and transparent polymer gels were observed while measuring the frictional force. The visualized frictional contact area due to the differences of refractive index and the frictional measurement data indicated that the local deformation by adhesion between gels and counter sample cause high friction resistant as a static friction coefficient.
\end{abstract}

\section{Introduction}

Friction and lubrication are one of the familiar physical phenomena and have been studied for a long time because of its importance in engineering. It is commonly known that the friction behavior of soft materials such as polymers and rubbers is different comparison with hard materials (Grosch 1963). Polymer gels are also typical soft materials and its frictional properties have been studied experimentally in systematic (Gong et al. 1997, 2001; Ren et al. 2015). In recent years, hydrogel's superior properties including frictional characteristic have been significantly focused in the fields of material and biomedical science due to the ability to hold large amount of water in the crosslinked polymer network which is considered as inherent

Hidemitsu Furukawa

furukawa@yz.yamagata-u.ac.jp

1 Department of Mechanical Systems Engineering, Graduate School of Science and Engineering, Yamagata University, 4-3-16 Jonan Yonezawa, Yamagata 992-8510, Japan

2 Mechanical Course, Department of Creative Engineering, National Institute of Technology, Tsuruoka College, 104 Sawada Inooka, Tsuruoka, Yamagata 997-8511, Japan beneficial properties of hydrogels (Green and Elisseeff 2015).

To date, a lot of functional gels have been reported and enhanced the possibilities of hydrogels as shown in follows, e.g., volume phase transition (Tanaka 1978), low frictional properties (Wada et al. 2013, 2018), shape memory gels (Kagami et al. 1996), self-healing gels (Kakuta et al. 2013; Sun et al. 2008), self-oscillating gels (Yoshida et al. 1996) and high strength gels (Okumura and Ito 2001; Haraguchi and Takehisa 2002; Gong et al. 2003; Sakai et al. 2008; Wang et al. 2010). These unique properties were realized by designing the ingenious polymer structures. Furthermore, the development of additive manufacturing method for hydrogels overcame the difficulty of free-forming and provided new possibilities as mechanical materials (Muroi et al. 2013; Shiblee et al. 2018, 2019). These fundamental and practical studies are expected to become the bases of the application such as a soft robot consisting of living body like soft and wet materials.

Double Network gels (DN gels) are one of the high strength gels having interpenetrating polymer networks consist of different two types polymers. Typical type of DN gels are prepared by two types of different gels which are electrolyte gel as first polymer network and neutral gel as 

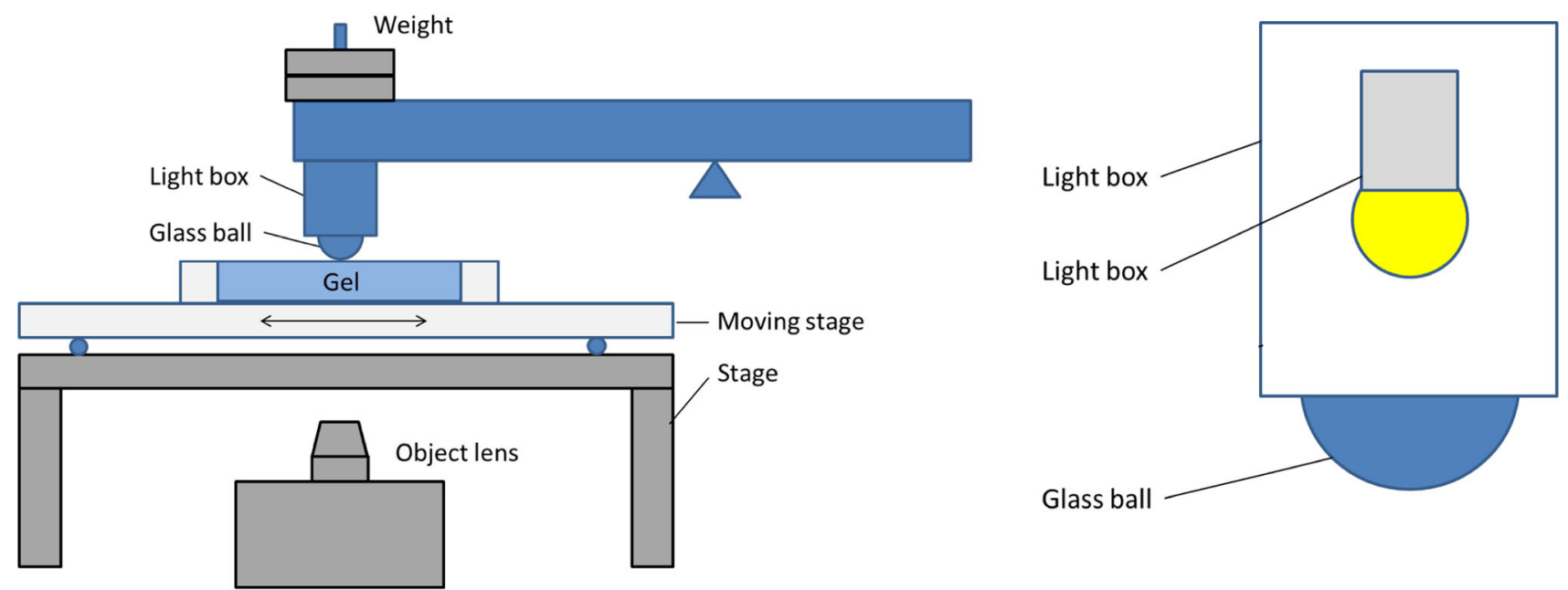

Fig. 1 The schematic depiction of measuring and observation system

second. Despite using weak gels as a material, the maximum breaking stress in compression test of well-designed DN gels reaches several $10 \mathrm{MPa}$ with high amount of water around $90 \%$. Besides, it is revealed that DN gels have lowfriction surface and it has 10-4 order of frication coefficient by a chemical modification of surface. Such mechanical properties shows the remarkable value which is equal to or greater than the joint cartilage in our living body. Consequently, DN gels are expected to be applied as soft and wet components in soft robotics.

In the study on friction, the observation of frictional surface have been played an important roles to understand its phenomena. There are many this kinds of researches being reported by using microscope, $\mathrm{X}$-ray photograph and infrared light, but few researches on the surface of polymer gels (Newby and Chaudhury 1998; Yamamoto et al. 2014; Nanao et al. 2015; Hase 2019). In terms of the friction of polymer gels, water layer in the interface contributes to the reduction of frictional resistance. The adsorption of surface polymer to the counter sample is dominant factor in low friction speed, however, the frictional coefficient decreases as the speed increases because the water is drawn into the frictional interface. In addition, the frictional behavior under the condition the contact region deformation occurs cannot be neglected for the practical use. When considering these points, it can be said that the direct observation of frictional behavior is important to understand polymer gel surface characteristic. Inhere, we report that the development of the microscopic observation system of frictional interface between polymer gels and a glass ball and show the results when water was not rich on the surface.



Fig. 2 The picture of observation instrument and the detail of measuring part

\section{Methods}

\subsection{Observation and measuring system designing}

We designed the instrument for the observation of dynamic friction interface between transparent polymer gels and a glass ball (developed jointly with Trinity-Lab inc.). The frictional interface pictures were taken macroscopically by the microscope camera (DP71, Olympus Corp.). The schematic depiction and pictures of the instrument are shown in Figs. 1 and 2 separately. The ball-on-disk type measurement instrument was arranged so as to straddle inverted microscope (IX71, Olympus Corp.) and to position the contact area exactly on the objective lens. This friction measurement part was designed based on conventional model ( $\mu \mathrm{V} 1000$, Trinity-Lab inc.). The balancing toy structure can apply arbitrary vertical load from 50 to $500 \mathrm{~g}$. The sample stage can move horizontal uniaxial direction at 
arbitrary velocity from 0.1 to $100 \mathrm{~mm} / \mathrm{s}$ with the maximum distance of $200 \mathrm{~mm}$. The load cell was built in the part of the balancing structure to measure the friction coefficient. The LED light source was incorporated inside the ball holder. A transparent glass balls (BK-7) were used as the counter sample toward hydrogels and a glass disk was fixed on the moving stage so that white light can pass through from the light source to an objective lens. The frictional surface pictures were taken as moving images with the microscope camera.

\subsection{Gel synthesis}

Double Network gels were prepared by two times synthesis processes of an electrolyte gel and a neutral gel. The 1st gels were synthesized by using 2-acrylamido-2-methylpropanesulfonic sodium salt (NaAMPS, Sigma-Aldrich Co. LCC.) as monomer, $N, N^{\prime}$-methylenebis-acrylamide (MBAA, FUJIFILM Wako Pure Chemical Corp.) as crosslinker, 2-oxoglutaric acid ( $\alpha$-keto, FUJIFILM Wako Pure Chemical Corp.) as UV initiator and pure water as solvent. The $4 \mathrm{~mol} \%$ of MBAA and $0.1 \mathrm{~mol} \%$ of $\alpha$-keto were added to $1 \mathrm{M}$ NaAMPS aqueous solution. After being bubbled with nitrogen gas for $15 \mathrm{~min}$, the precursor solution was poured into the mold which was prepared by sandwiching the silicone spacer of $1 \mathrm{~mm}$ thickness between two pieces of glass plates. The pre-gel solution was irradiated with ultraviolet rays for $1 \mathrm{~h}$ at room temperature $30{ }^{\circ} \mathrm{C}$ in UV curing box (Peak wavelength $352 \mathrm{~nm}$; intensity $0.1 \mathrm{~mW}$; TOSHIBA Corp.). Then 1 st gel was swollen with 2 nd gel solution for $48 \mathrm{~h}$ to reach the equilibrium swelling. The 2 nd gel solution was prepared by stirring $0.02 \mathrm{~mol} \%$ of MBAA and $0.1 \mathrm{~mol} \%$ of $\alpha$-keto into $1 \mathrm{M}$ of $N, N$-dimethylacrylamide (DMAAm, Tokyo Chemical Industry Co., Ltd.) aqueous solution and the solution was bubbled with nitrogen gas for $15 \mathrm{~min}$. The swollen gel was sandwiched between a couple of glass plates and the polymerization was induced by UV irradiation for $1 \mathrm{~h}$ in the constant temperature $30^{\circ} \mathrm{C}$. After the two times gelation, gels were swollen with pure water for $48 \mathrm{~h}$.

\subsection{Measurement and observation setting}

DN gel sheet was adsorbed on the moving glass stage by their natural adsorbability when water on the surface lost not to slip between the stage and the opposite side of measuring surface. Here, two types of surface, dry surface and water rich surface, were prepared as shown in Fig. 3. An objective lens magnification was 4 and the stage height of measurement part was adjusted to have appropriate distance to focus on the frictional interface between gel and glass ball. The vertical load was $100 \mathrm{~g}$ and the sliding velocity was $10 \mathrm{~mm} / \mathrm{s}$ in this experiment.
Fig. 3 The experiment settings of polymer gels on the stage; wiped surface (left) and water rich surface (right)
Fig. 4 The contact areas between hydrogels and glass ball during the frictional measurement, the initial position (a) and the position at the time $0.09 \mathrm{~s}$ after the measurement started (b). The outline of contact area could be confirmed clearly. The displacement of contact area caused by frictional resistant
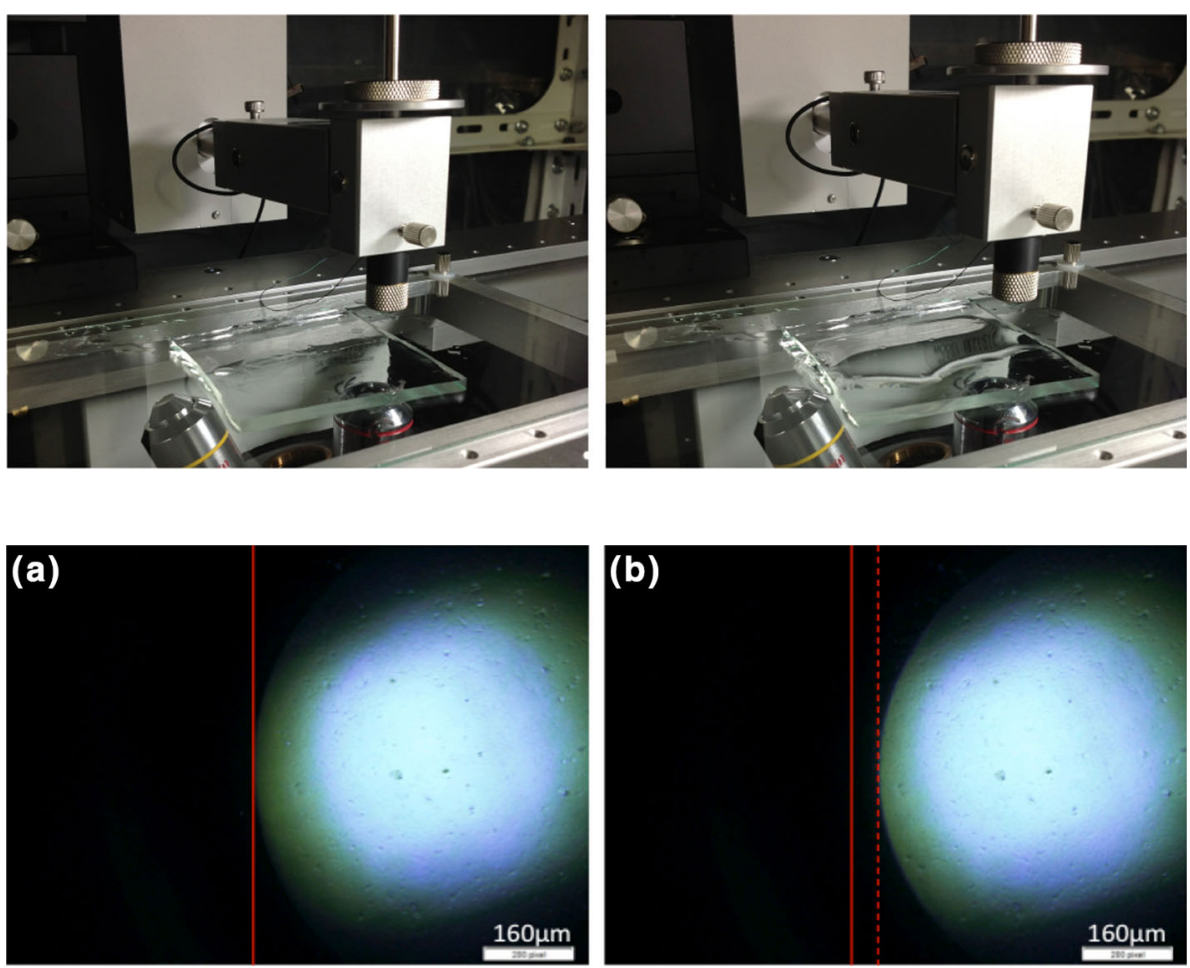


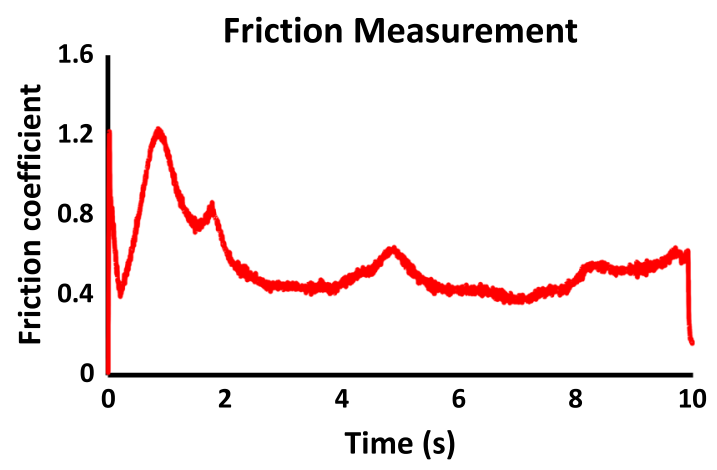

Fig. 5 The frictional measurement result during the experiment shown in Fig. 4. In the beginning of the measuring, the result showed high static ftiction coefficient with the observation of the displacement of contact area shown in Fig. 4

\section{Results}

When the surface was filled with water, the differences between the frictional surface and non-contact area were not recognized because of the less difference in the reflective index between DN gels, glass ball and water. Figure 4 shows the frictional measurement after wiping water on the surface. The parameter of microscope image such as exposure time and white balance were calibrated so that the images can show the contact area clearly. Figure $4 \mathrm{a}$ shows the initial position of measurement. The contact area was distinctly visible in air condition and the moving pictures made it possible to confirm the displacement of contact area behind the travelling direction as shown broken red line in Fig. 4b. This displacement occurred immediately by the static friction at the beginning of measurement and the contact area remained in the backward during the measurement. It is considered that the deformation in frictional measurement cause the displacement of contact area by the frictional resistance. Figure 5 shows the raw frictional measurement data carried out at the same time as the observation. The frictional coefficient showed the static friction coefficient of 1.23 . The dry surface means there was the high adhesion between the surface of gels and a glass ball and the softness of gels and that cause the unstable coefficient value shown in Fig. 5.

\section{Discussion}

In this experiment, the frictional interface was observed when water was not rich on the friction surface. On the other hand, the contact area between gel and glass were not observed because of the similarity of reflective index. To visualize the wet surface is a future consideration, and it is potentially resolvable by using optical glasses or lubricant with high transparency and a high reflective index. The colored lubricant might help to figure the contact area and water layer. However, it is indicated that the deformation by adhesion at friction surface induced the frictional coefficient high. The unstable friction coefficient as shown in Fig. 5 was seen in the measurement of well lubricated condition. In the future work, the measurement will be carried out with various speed and weight and we would like to clarify the frictional behavior from the observation of the boundary lubrication and the hydrodynamic lubrication in polymer gel friction.

\section{Conclusions}

The microscopic observation system of the friction surface of polymer gels was developed by combining inverted microscope and specially arranged ball-on-disk friction measuring machine. The contact area during the friction under deformation condition between hydrogels and a glass ball was observed at the same time as the frictional measurement. When the surface was not filled with water, the results showed that hydrogels were deformed by the frictional force and the displacement of contact area was observed during frictional measurement.

Acknowledgements This study has been partly supported by a Grantin-Aid for Scientific Research (Category A, Project no. JP17H01224, 17H01224) from the Japan Society for the Promotion of Science (JSPS), Green Network of Excellence (GRENE) (Green Tribology Innovation Network) from the Ministry of Education, Culture, Sports, Science and Technology (MEXT), the Center of Innovation (COI) program (Project No. JPMJCE1314) from the MEXT and the Japan Science and Technology Agency (JST), Cross-ministerial Strategic Innovation Promotion Project (SIP) 1st/2nd from the Cabinet Office and the Program on Open Innovation Platform with Enterprises, Research Institute and Academia (OPERA) (Project no. JPMJOP1844, JPMJOP1614) from the JST.

\section{Compliance with ethical standards}

Conflict of interest The authors declare that they have no conflict of interest.

Open Access This article is distributed under the terms of the Creative Commons Attribution 4.0 International License (http://creative commons.org/licenses/by/4.0/), which permits unrestricted use, distribution, and reproduction in any medium, provided you give appropriate credit to the original author(s) and the source, provide a link to the Creative Commons license, and indicate if changes were made.

\section{References}

Gong JP, Higa M, Iwasaki Y, Katsuyama Y, Osada Y (1997) Friction of gels. J Phys Chem B 101:5487-5489 
Gong JP, Kagata G, Iwasaki Y, Osada Y (2001) Surface friction of polymer gels 1. Effect of interfacial interaction. Wear 251:1183-1187

Gong JP, Katsuyama Y, Kurokawa T, Osada Y (2003) Double network hydrogels with extremely high mechanical strength. Adv Mater 15(14):1155-1158

Green JJ, Elisseeff JH (2015) Mimicking biological functionality with polymers for biomedical applications. Nature 540(7633):386-394

Grosch KA (1963) The relation between the friction and viscoelastic properties of rubber. Proc R Soc Lond Ser A Math Phys 274(1356):21-39

Haraguchi K, Takehisa T (2002) Nanocomposite hydrogels: a unique organic-inorganic network structure with extraordinary mechanical, optical, and swelling/de-swelling properties. Adv Mater 14(16): 1120-1124

Hase A (2019) Visualization of the tribological behavior of graphite in cast iron by in situ observations of sliding interfaces. Tribol Int 138:40-46

Kagami Y, Gong JP, Osada Y (1996) Shape memory behaviors of crosslinked copolymers containing stearyl acrylate. Macromol Rapid Commun 17(8):539-543

Kakuta T, Takashima Y, Nakahata M, Otsubo M, Yamaguchi M, Harada A (2013) Preorganized hydrogel: self-healing properties of supramolecular hydrogels formed by polymerization of hostguest-monomers that contain cyclodextrins and hydrophobic guest groups. Adv Mater 25:2849-2853

Muroi H, Hidema R, Furukawa H (2013) Development of optical 3D gel printer for fabricating free-form soft and wet industrial materials and evaluation of printed double-network gels. J Solid Mech Mater Eng 7(2):163-168

Nanao H, Hishi Y, Shizuku T, Takiwatari K, Mori S (2015) Direct observation of lubricant components between wire and diamond die for wire drawing with a micro-FTIR. Technol Lett 60:12

Newby BZ, Chaudhury MK (1998) Friction in adhesion. Langmuir 14(17):4865-4872

Okumura Y, Ito K (2001) The polyrotaxane gel: a topological gel by figure-of-eight cross-links. Adv Mater 13(7):485-487

Ren HY, Mizukami M, Tanabe T, Furukawa H, Kurihara K (2015) Friction of polymer hydrogel studied by resonance shear measurements. Soft Matter 11(31):6192-6200
Sakai T, Matsunaga T, Yamamoto Y, Ito C, Yoshida R, Suzuki S, Sasaki N, Shibayama M, Chung U (2008) Design and fabrication of a high-strength hydrogel with ideally homogeneous network structure from tetrahedron-like macromonomers. Macromolecules 41(14):5379-5384

Shiblee MDNI, Ahmed K, Khosla A, Kawakami M, Furukawa H (2018) 3D printing of shape memory hydrogels with turnable mechanical properties. Soft Matter 14:7809-7817

Shiblee MDNI, Ahmed K, Kawakami M, Furukawa H (2019) 4D printing of shape-memory hydrogels for soft-robotic functions. Adv Mater Technol 5:545. https://doi.org/10.1002/admt. 201900071

Sun TL, Kurokawa T, Kuroda S, Ihsan AB, Akasaki T, Sato K, Haque MA, Nakajima T, Gong JP (2008) Physical hydrogels composed of polyampholytes demonstrate high toughness and viscoelasticity. Nat Mater 12(10):932-937

Tanaka T (1978) Collapse of gels and the critical endpoint. Phys Rev Lett 40:820

Wada M, Hidema R, Chiba T, Yamada K, Yamada N, Gong J, Furukawa H (2013) Surface and bulk mechanical properties of soft and wet materials. J Solid Mech Mater Eng 7(2):228-234

Wada M, Yamada K, Kameyama T, Yamada N, Yoshida K, Saito A, Makino M, Khosla A, Kawakami M, Furukawa H (2018) Electric control of friction on surface of high-strength hydrogels. Microsyst Technol 24:639-646

Wang Q, Mynar JL, Yoshida M, Lee E, Lee M, Okuro K, Kinbara K, Aida T (2010) High-water-content mouldable hydrogels by mixing clay and a dendritic molecular binder. Nature 463(7279):339-343

Yamamoto T, Kurokawa T, Ahmed J, Kamita G, Yashima S, Furukawa Y, Ota Y, Furukawa H, Gong JP (2014) In situ observation of a hydrogel-glass interface during sliding friction. Soft Matter 10:5589-5596

Yoshida R, Takahashi T, Yamaguchi T, Ichijo H (1996) Selfoscillating gel. J Am Chem Soc 118:5134-5135

Publisher's Note Springer Nature remains neutral with regard to jurisdictional claims in published maps and institutional affiliations. 